\title{
DOES HERD BEHAVIOUR ARISE EASIER UNDER TIME PRESSURE? EXPERIMENTAL APPROACH
}

\author{
Lubomír Cingl*
}

\begin{abstract}
:
In this paper I explain individual propensity to herding behaviour and its relationship to time-pressure by conducting a laboratory experiment. I let subjects perform a simple cognitive task with the possibility to herd under different levels of time pressure. In the main treatments, subjects had a chance to revise their decision after seeing decisions of others, which I take as an indicator of herding behaviour. The main findings are that the propensity to herd was not significantly influenced by different levels of time pressure, although there could be an indirect effect through other variables, such as the time subjects spent revising the decision. Heart-rate significantly increased over the baseline during the performance of a task and its correlation to the subjectively stated level of stress was positive but very weak, which suggests that time pressure may not automatically induce stress but increase effort instead.
\end{abstract}

Keywords: herding, experimental economics, heart rate measurement, personality traits

JEL Classification: C25, C91, D03, D80

\section{Introduction}

The main goal of this paper is to discover the effect of time pressure on the individual propensity to herd, and the form of this effect in relationship to varying levels of time pressure and personal characteristics. Revealing the underlying nature of the relationship may be important in the explanation of real-life phenomena such as fads, fashion, but also panic in financial markets. I expect the time-constraint to induce stress reaction which in turn should influence the individual decision-making process (Lundberg, 1993). One of the products of the altered decision-making may be that the subjects are more likely to imitate other's behaviour. The underlying mechanism depends on the accepted theoretical explanation of herding as there have been two main approaches proposed: the informational and the behavioural approach. A theoretical synthesis of these two approaches has already been made in Cao and Hirshleifer (2000) and this experiment does not try to resolve the duality between them as has already been made in Baddeley et al. (2007), but rather it focuses on the relationship of time pressure and herding, which has been so far omitted. I hypothesize that the occurrence of herding

* IES FSV Charles University, Prague, Opletalova 26, CZ - 11000 Prague 1 (lubomir.cingl@gmail.com). The funds were provided by Charles University GAUK Grant No. 59110 and J\&T Bank, a.s. The research also was gratefully supported by the possibility of using the heart-rate monitors of the Laborator sportovni motoriky FTVS UK, by Dr. Josef Horčic. 
and information cascades is more frequent under more severe time pressure. In this experiment I also show the important characteristics driving the decisions of subjects whether or not to conform to publicly available information and the interaction with time pressure. I expect emergence of various types of agents' behaviour, which is discussed in more detail below: agents with low task-specific confidence and high score in the personality trait Agreeableness are expected to be more likely to follow the herd whereas subjects with high confidence and high scores in Conscientiousness are expected to be less likely to follow the herd.

The task that subjects were to solve appeared first in Falk et al. (2006) and was modified to meet needs of this experiment. The task was to count the number of zeros in a table of 400 symbols, where only ones and zeros occurred. Performance was rewarded by a fixed-payment for accuracy and by decreasing time-dependent pay-off. In the treatment condition the subjects, after setting their first guess of the correct number, had an opportunity to see the first guesses of faster subjects, and after this to change their guess to another number. Subjects could decide first whether or not to see the information about the others' results and then whether or not to change their guess. If a subject looked at information about the others' results and changed her guess, it is used as $0 / 1$ proxy for the occurrence of herd behaviour, which is then modelled as an explained variable by using logistic regression. They performed the task under three different types of time-pressure, which is then used as a set of $0 / 1$ explanatory variables.

The behavioural approach to herding suggests that it is an innate characteristic of a human species resulting in preferences for conformity. This implies that the decision about whether or not to follow the herd is an instinctive response and as such it should be very quick and the probability to herd should depend on personal characteristics. If this is the case, the varying levels of time pressure should not influence the propensity to herd as decision to herd needs always only very little time to decide upon. Personal characteristics are tracked and controlled for by using the standardized psychometric protocols IPIP-NEO from Goldberg (2010). Tracking of personal characteristics is important for other reasons, e.g. as stress can have a different impact on the performance of people with different attitudes to risk as in Cadsby et al. (2009). Baddeley et al. (2010) found positive association of herding with personality traits associated with risk-taking, namely impulsivity and venturesomeness.

The (Bayesian) informational approach on the other hand suggests that herding is a result of a rational use of additional information extracted from the spotted behaviour of others. As such, when having not enough time to extract the information both from one's own resources and from the information pool of others, it will depend on what information source is perceived as being more reliable. Maule and Edland (2000) provide a very interesting review of the effects of time-pressure on individual decision making, which as they conclude has been mainly ubiquitous - similarly to other studies. Rieskamp and Hoffrage (2008) as well as Payne et al. (1996) show that the main effect of time-constraint on information-processing is that participants process information faster and with higher selectivity of important facts. Kocher and Sutter (2006) in the framework of an 
experimental beauty-contest game found that the convergence to equilibrium is faster and the pay-offs are higher in the low pressure treatment than in the high time pressure, however, during the high pressure treatment the quality of decision making does not decrease. One form of herding resulting from the rational use of information is the so called information cascade. Information cascades were first comprehensively described and analysed by Bikhchandani et al. (1992), Banerjee (1992) and Welch (1992), and the first most widely respected test of this claim was carried out by Anderson and Holt (1997).

Baddeley et al. (2007) conclude that both approaches have some merit and none of them can be used exclusively. Baddeley et al. (2010) suggest developing a neuroeconomic behaviour model with emphasized dual processing and consilience of both the mentioned approaches.

\section{Methodology}

I conducted a computerized ${ }^{1}$ laboratory experiment to test the theoretical predictions which I summarize in Section 3. Participants of the experiments had to complete a task of counting zeros from a sheet of 400 symbols under different levels of time constraint. The experiment consisted of six experimental sessions in two days plus one pilot session that was used for parameter calibration. There were three different within-subject treatments: in the first treatment the subjects were not restricted by time and had two tasks only to practice. In the second treatment three levels of time pressure were introduced, which was represented by a strict ${ }^{2}$ time constraint and a time-dependent bonus to motivate the subjects to be fast. The third treatment introduced the core idea of the opportunity to look at the guesses of others who were faster than the subject and then to revise the original guess to a new one, so in effect there was a counting part and a revision part of the task.

The time pressure had to be imposed both on the counting part as well as on the revision part of the task: ${ }^{3}$ time pressure in the counting part served as a generator of uncertainty about one's private information; and time pressure in the revision part was expected to cause effect on individual propensity to herd. If there had been no time pressure in the counting part of the task, everybody would have reached very precise private information and thus would have had no incentive to revise it by taking inspiration from others. Apart from this, exposure to a time pressure induces physiological stress reaction only after some time (Kemeny, 2003) so having a shortage of time only for the revision would have had no impact on the decision.

In the revision part of the task a subject was able to observe only the results of subjects

1 Program used was the Z-TREE (Fischbacher, U. (2007), "Z-TREE: Zurich Toolbox for Ready-made Economic Experiments.” Experimental Economics, Vol. 10, pp. 171-178.)

2 Strict in the sense that if a subject ran out of time, received zero ECU.

3 There was one time constraint common for both the counting and the revision parts, so the time was still running out during both counting as well as during revising one's decision. 
who were faster than her, which corresponds to situations in the real world, where we almost always observe only actions that were made before our decision. This has certainly had implications for individual strategy concerning the revisions: the faster subjects could have had worse quality of public information, but on the other hand they could have had better individual abilities to solve such task. Strategically thinking subjects usually observed that there was a stable portion of "honest" subjects who always counted until the end and their estimate was reliable, and thus it was worth waiting for them and copying their results.

Heart-rate was used as a proxy of endured stress and it was measured by heart-rate monitors Polar R800 with a precision of one second. The individual difference of the average heart-rate during the task minus the base-level is used as an explanatory variable in the regression analysis. Heart rate increases are correlated with endured psychosocial stress as was shown e.g. by artificial induction of stress in Trier Social Stress Test (Kirschbaum et al., 1993) and is generally considered to be a sign of increased body activity. The caveat is that increased heart-rate may be a result of other stimuli than stress, which imposes limitation to the explanation of the result. Moreover, for precise measurement of stress this should be combined with other measures like concentration of cortisol in saliva or systolic blood pressure. However, the response of HPA axis to the endured stress in case of release of cortisol is not immediate and its administration is distracting and relatively costly. Measurement of blood pressure would significantly prolong the experiment and it would not be possible to administer during the task, therefore I decided to use only heart-rate as a measure of stress with the known limitations of this approach.

In this context I define the occurrence of herding as a situation when a participant used information from seeing the guesses of the other participants. This $0 / 1$ variable is meant to be the observable outcome of an unobservable probability to herd, which is the main variable of interest. The subjects could choose whether or not to see the public information (see the scheme of decision-making flow in the Chart 1).

Chart 1

Scheme of Decision-making Process after Setting the First Guess

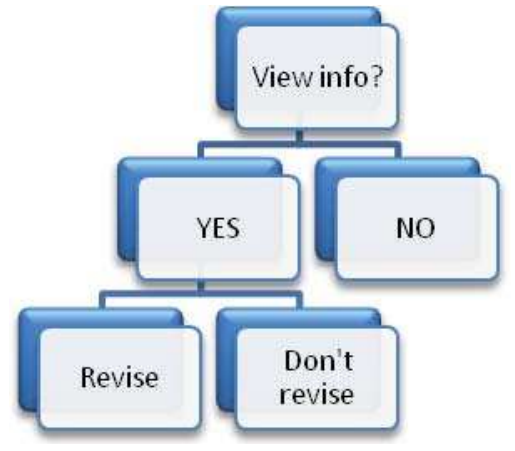

Source: author. 


\subsection{Task: Counting Zeros}

The participants performed a simple cognitive effort task introduced in Falk et al. (2006), which was supposed not to require previously earned skills or any innate cognitive abilities or learning effect (see Chart 2)

This task was also designed not to involve any emotions and only positive pay-offs were possible to eliminate loss-aversion. The signal imperfection is induced by utilizing the subjects' inability to cope with the situation in being under time-pressure. Participants were required to count a correct number of zeros from a table of 400 symbols (zeros and ones only) that appeared on the screen. The numbers are randomly generated from a uniform distribution with variability large enough so that accurate guessing is highly improbable. Each participant was supposed to solve eleven tasks in total, including the practice session. After counting the number of zeros, participants were supposed to enter their estimated number (guess) into a field on the screen.

Chart 2

Task Screen in Treatment 1

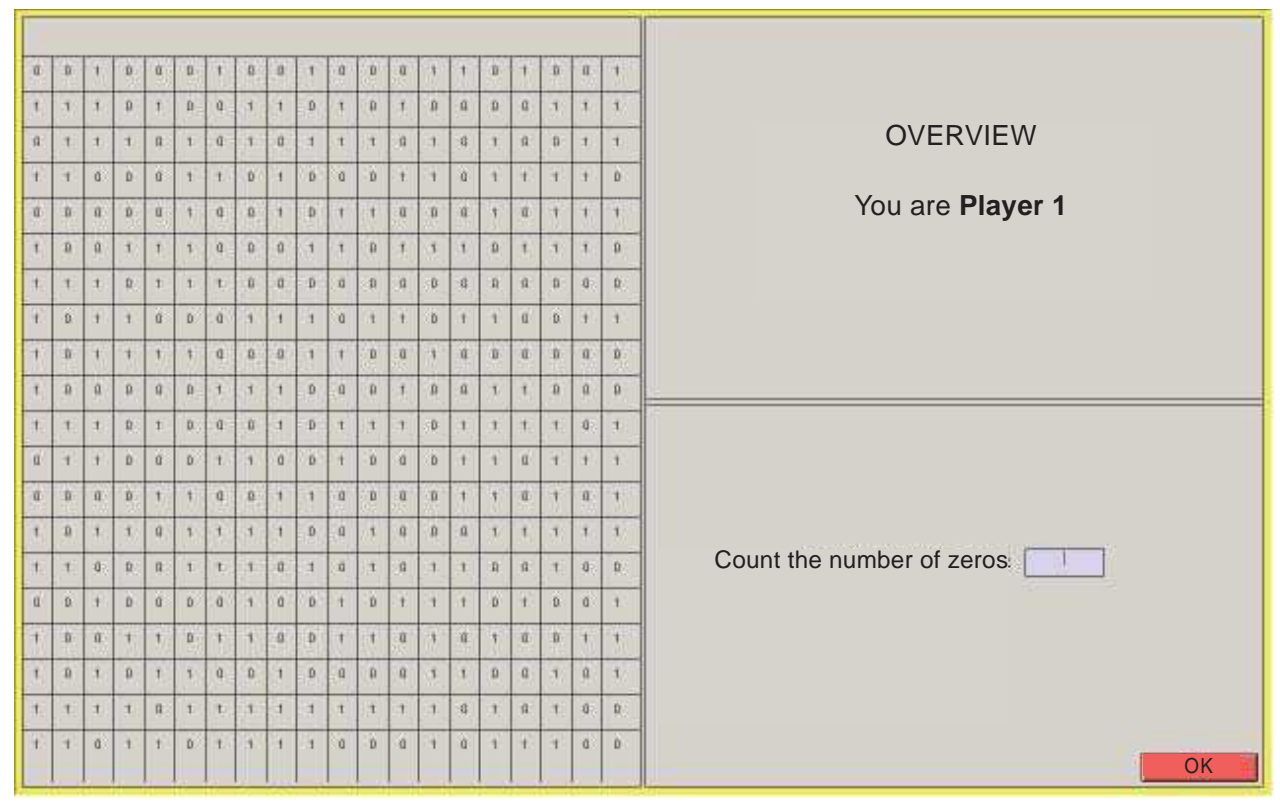

Source: author.

\subsection{Pay-off function}

Pay-off function consisted of a fixed part and a time-dependent part. Similarly as in Falk et al. (2006), participants were paid fixed amount of $100 \mathrm{ECU}(2 €)$ per task if counted exactly, $80 \mathrm{ECU}$ if in the range of $+/-1$ or $40 \mathrm{E} \mathrm{CU}$ if in range $+/-2$. The size of the 
time-dependent part was different with each level of time pressure (see Table 1). The time limit was binding in the sense that if the task was not completed in the given time, participant got zero ECU in total for the given task. Also the precision of the guess was binding such that if a participant missed the correct number of zeros by more than two, she received zero from both fixed amount as well as from the time dependent bonus. The fixed part of the payment per task is the motivation for a subject to count accurately while the time-dependent bonus part makes the subject count as quickly as possible. Being fast and precise is normally a stressful and demanding on concentration. All subjects are under the same level of time pressure at the time, so the individual performance relative to others should stay the same and the beliefs about other subjects and the probability of their success should not change with different levels of time pressure.

Table 1

Pay-off Function Parameters

\begin{tabular}{|l|c|c|c|}
\hline $\begin{array}{l}\text { Level of time } \\
\text { pressure }\end{array}$ & Time limit & $\begin{array}{c}\text { Time-dependent part } \\
\text { (start value) }\end{array}$ & $\begin{array}{c}\text { Factor of decreasing } \\
\text { (per second) }\end{array}$ \\
\hline Low & $150 \mathrm{~s}$ & $400 \mathrm{ECU}$ & $-3 \mathrm{ECU}$ \\
\hline Medium & $130 \mathrm{~s}$ & $500 \mathrm{ECU}$ & $-4 \mathrm{ECU}$ \\
\hline High & $100 \mathrm{~s}$ & $600 \mathrm{ECU}$ & $-5 \mathrm{ECU}$ \\
\hline
\end{tabular}

Source: author.

\subsection{Sample description}

The experiment took place at the premises of CERGE-EI and was attended by 90 participants in total. A majority of participants were undergraduate students from Prague - mostly Czechs (77.8\%) followed by Slovaks (12.2\%) and other nationalities (10\%). The participants were mainly males $(62.2 \%)$, the most common field of study was economics and business (75\%) and the median age was 22. Participants were recruited using an online database of CERGE-EI and were paid privately at the end of the experiment, the average payment was CZK 350 (app. 13.5 €) including a guaranteed show-up fee of CZK 150 (app. $6 €$ ). The average payment was about double the average hourly salary in region. In total, the experiment lasted less than 2 hours. Due to the low variation in age, education and nationality I did not use these to be control variables in the model, however, it may be important in other settings.

\subsection{Organization of the experiment}

The timeline of the experiment is summarized in Table 2. Before the start of the experiment, the heart-rate monitors were attached and during the rest of the experiment the heart-rate of the participants was recorded. After reading the instructions aloud and explaining them in detail, subjects were asked a few questions to check their understanding of the rules. The participants went through three main parts of the experiment that were based on the 
task described above. The first part included the first treatment to familiarize subjects with the task; the second and the third parts included the second and the third treatments, respectively. Each participant was supposed to solve two tasks in the first treatment, three tasks in the second treatment and six tasks in the third treatment. Participants were informed before each task about the level of time pressure, the time limit for the task and the bonus they could get. This information was provided on a separate introductory screen. Participants saw their pay-offs from the task always on a summary screen after each task and this screen also included the cumulative pay-off from the treatment. At the end of each task, the participants had to answer a question on their subjective perception of the pressure they were under (as in Svenson and Benson, 1993). This result is then compared to the data from the heart-rate monitors. Prior to the end of the experiment, the participants had to fill out a questionnaire. After this they were asked to stay a few minutes at rest which was necessary to establish a reference level for the heart rate.

Table 2

Timeline of the Experiment

\begin{tabular}{|c|c|}
\hline \multicolumn{2}{|c|}{ Subjects arrive to the lab } \\
\hline \multicolumn{2}{|c|}{ Heart-rate monitors attached } \\
\hline \multicolumn{2}{|c|}{$\begin{array}{l}\text { Reading instructions aloud } \\
\text { control questions }\end{array}$} \\
\hline \multicolumn{2}{|c|}{$\begin{array}{l}\text { First part - acquainting with the task } \\
2 \text { tasks to solve }\end{array}$} \\
\hline \multicolumn{2}{|c|}{$\begin{array}{l}\text { Second part - introducing time pressure } \\
\qquad 3 \text { tasks to solve }\end{array}$} \\
\hline \multicolumn{2}{|c|}{$\begin{array}{l}\text { Risk-preferences protocol - lottery task } \\
\text { Task-confidence }\end{array}$} \\
\hline \multicolumn{2}{|c|}{$\begin{array}{l}\text { Third part } \\
6 \text { tasks to solve under varying levels of time pressure } \\
\text { Possibility to see public information and revise a decision }\end{array}$} \\
\hline \multicolumn{2}{|c|}{$\begin{array}{l}\text { Questionnaire } \\
\text { personality profile - traits } \\
\text { Personal characteristics }\end{array}$} \\
\hline $\begin{array}{l}\text { Sitting } \\
\text { payol }\end{array}$ & $\begin{array}{l}\text { quiet } 5 \text { mins } \\
\text { ut \& leave }\end{array}$ \\
\hline
\end{tabular}

Source: author. 


\section{Test of task-specific confidence, risk-preferences protocol and personality traits}

Before the third part of the experiment, the participants were asked a question on their judgment about their respective performances ${ }^{4}$ in the task in order to measure how confident the participants felt. The answer ranged from one to five with one being the top $20 \%$ and five being the bottom $20 \%$ and it enters the model in the form of the variable SelfConfidence. After they had finished this, the participants were asked to fill out a separate sheet of paper with a "lottery card" based on Dohmen et al. (2009) to find out their attitude to risk. To capture the personality profile of participants, I used the "Big Five" factors that are Openness to Experience, Conscientiousness, Extraversion, Agreeableness and Neuroticism. Each factor represents a summary of a large number of specific personality characteristics (Goldberg, 2010).

\subsection{Hypotheses description}

\section{Effect of time pressure on decision making}

Generally speaking, if participants were perfectly rational, they would neither fail in the task nor would seek information about the decisions of other participants. If we relax this assumption by assuming that individual decision-making is based on individual bounded rationality, then we should expect a negative monotonic relationship between the level of time pressure and performance in the task. Gilbert and Kogan (2005) show that learning from others has an impact mostly on worse players, who tend to improve not only results, but also decision making processes. The reasoning should be straightforward: the less time the subject has for completing the task (which corresponds with a higher level of time pressure) the less precise her private information gets and the more relevant it is to seek for and use the public information. Rieskamp and Hoffrage (2008) show however that when people are under increased time-pressure, they tend to process information faster and focus more selectively on the more important information. The effect of time pressure on herding will therefore depend on the individual assessment of whether the public information may or may not be useful.

Hypothesis 1: Herding is more frequent under higher time pressure. Time pressure is a relevant variable in explaining the probability of herding.

\section{Heart rate}

Heart rate is the frequency of the contractions of the heart muscle and its unit of measurement is frequency per minute. Changes in heart rate refer to higher levels of arousal, which are often somatically mediated, which suggests that when the heart-rate increases, the body is in a state of increased awareness, such as stress. However, heart-

4 Exact wording of the question: "Please try to guess, in which part of the distribution of results you are (i.e. if you think, that you are in the top $20 \%$, please click on the "Top $20 \%$ ", which means how close you are to the top)." 
rate as a psycho-physiological variable is a rather rough measure of stress as stated in Lo and Repin (2001).

Hypothesis 2: Stress induced by the time pressure causes the individual's heart rate to be significantly different from the base level during the performance and is positively correlated with the subjectively stated level of stress.

\section{Model Description}

Binary variable InfoUsed was defined as one for the situation when a subject changed her decision after being confronted with the decisions of others, and zero otherwise. These two outcomes are mutually exclusive and I assume that they arise with probability $\operatorname{Pr}[$ InfoUsed $]$ and $1-\operatorname{Pr}[$ InfoUsed $]$, respectively. The two standard binary outcome models are the logit and probit models and the parameter estimation is carried out in the maximum-likelihood fashion (for details see Cameron and Trivedi, 2010). As I could not be sure if the distribution of choices follows normal distribution, I decided to use standard logistic regression ${ }^{5}$ with robust standard errors to account for interpersonal/intragroup correlations (Greene, 2002). Apart from that, binary choice tasks with social interaction give statistical models that are mathematically equivalent to logistic discrete choice (Baddeley, 2007 or Brock and Durlauf, 2000). The model for explaining the probability of herding, or in other words the binary variable InfoUsed given the information set a subject had available for her decision, stands as follows:

$$
\begin{gathered}
\log \left(\frac{\operatorname{Pr}[\text { InfoUsed }]}{1-\operatorname{Pr}[\text { InfoUsed }]}\right)=\beta_{1}+\beta_{2} \text { SelfConfidence }+\beta_{3} \text { TimeLeft }+\beta_{4} \text { TP } P_{\text {Medium }}+ \\
\beta_{5} T P_{\text {High }}+\beta_{6} O+\beta_{7} C+\beta_{8} E+\beta_{9} A+\beta_{10} N+\beta_{11} \text { SubjectiveStress }+\beta_{12} \text { Female }+ \\
\beta_{13} C E+\beta_{14} \text { RiskAverse }+\beta_{15} \text { InTotProf }+\beta_{16} \text { ObjectiveStress }+\beta_{17} \text { ScreenInfo }+ \\
\beta_{18} \text { GuesSSimilar }+\beta_{19} \text { TimeDeciding }+\epsilon_{i}
\end{gathered}
$$

I further assume that is normally distributed white noise stochastic term with zero mean and finite variance. To test the individual significance of each explanatory variable, I use z-test. To test the significance of the overall model I use the likelihood ratio test and the estimation procedure is implemented in the statistical package Stata 11.

\subsection{Variables description}

In the model specification, three groups of variables are incorporated: the first group represents the information that was on the screen with the public information, the second group represents the individual personality type and the third group contains other task characteristics that may be important for making the decision.

5 I also performed a robustness check of the results by carrying out an estimation of the same model by probit and linear probability model to find out that the results were almost equivalent across the three techniques, so I do not report it in this paper. 


\section{Dependent variable: InfoUsed}

The dependent variable InfoUsed was set equal to one if a subject adjusted her original estimate after seeing the table with the information about the decisions of others and zero otherwise.

\subsubsection{Independent variables}

\section{Time variables: TimeLeft, TimeDeciding}

Variable TimeLeft is the number of second participants had on the screen when they entered their original estimate. A majority of subjects did not have much time to waste so if they had it, they would invest it wisely. On the other hand, if already looking at the results of others, the total time they had left should already be irrelevant if it was above a certain threshold either there was useful info or less useful info, but the time to switch the estimate or to go further without switching was not dependent on the total time the subjects had.

Another explanatory dimension of time can be hidden in the time which subjects spent on the screen with the public information. Intuitively, because they were under time pressure, they must have decided fast whether to use the information and change the value, or to go further without changing the value, as described above. Had they decided to change their estimate, they had to think of the new value, which is already a deliberative process and needs more time, so the variable TimeDeciding, which indicates the time the subjects spent on the screen with the public info, is expected to be positively associated with the InfoUsed.

\section{Time Pressure indicator}

The exogenously set level of time pressure (low/medium/high) the subjects endured during the task is indicated by $0 / 1$ dummy variables. It enters the regression as a set of two variables TP_Medium and TP_High ${ }^{6}$ To test Hypothesis 1, these variables should be significant in the explanation of probability to herd, especially when indicating the "high" level of time pressure: the variable $T P \_H i g h=1$. The expected sign should be positive as stated in the Hypothesis 1.

\section{Stress variables}

The stress induced by the time pressure should also be an important variable and as part of Hypothesis 1 it should positively influence the probability of herding - InfoUsed. There appear two measures of it: the subjectively stated level of stress SubjectiveStress ${ }^{7}$ and the difference of the average level of heart-rate during the task to the base-line heart rate ObjectiveStress.

6 Time_Pressure_Medium and Time_Pressure_High. Due to perfect collinearity this brings, indicator of the treatment with low time pressure, TP_Low, must have been omitted

7 Participants had to state their subjectively perceived level of stress after each task on the scale from 1 to 10 with 1 being the least and 10 being the most stressful moment. 


\subsubsection{Control independent variables}

\section{Personality traits: OCEAN}

Following Baddeley et al. (2007), I expect that some individuals with certain personality characteristics will be more prone to follow behaviour of others, so I control for this by measuring the "Big Five" dimensions which is a standard psychological tool for assessment of personality traits. From the standardized battery of 50 scale questions I compute the scores for each of the five dimensions which I then put into the regression. See the summary of the dimensions in Table 3.

Table 3

The "Big Five" Domains and Their Facets

\begin{tabular}{|c|c|c|}
\hline Factor & Facets & Definition of a factor \\
\hline $\begin{array}{l}\text { I. Openness to } \\
\text { Experience }\end{array}$ & $\begin{array}{l}\text { Fantasy, Aesthetics, Feelings, } \\
\text { Actions, Ideas, Values }\end{array}$ & $\begin{array}{c}\text { The degree to which a person needs } \\
\text { intellectual stimulation, change, and } \\
\text { variety. }\end{array}$ \\
\hline II. Conscientiousness & $\begin{array}{c}\text { Order, Dutifulness, Achievement } \\
\text { striving, Competence, } \\
\text { Self-discipline, Deliberation }\end{array}$ & $\begin{array}{l}\text { The degree to which a person is } \\
\text { willing to comply with conventional } \\
\text { rules, norms, and standards. }\end{array}$ \\
\hline III. Extraversion & $\begin{array}{l}\text { Warmth, Gregariousness, } \\
\text { Assertiveness, Activity, Excitement } \\
\text { seeking, Positive emotions }\end{array}$ & $\begin{array}{l}\text { The degree to which a person needs } \\
\text { attention and social interaction. }\end{array}$ \\
\hline IV. Agreeableness & $\begin{array}{c}\text { Trust, Straightforwardness, } \\
\text { Altruism, Compliance, Modesty, } \\
\text { Tender-mindedness }\end{array}$ & $\begin{array}{c}\text { The degree to which a person needs } \\
\text { pleasant and harmonious relations } \\
\text { with others. }\end{array}$ \\
\hline $\begin{array}{l}\text { V. Neuroticism } \\
\text { (Emotional Stability) }\end{array}$ & $\begin{array}{l}\text { Anxiety, Angry hostility, Depression, } \\
\text { Self-consciousness, Impulsiveness, } \\
\text { Vulnerability }\end{array}$ & $\begin{array}{l}\text { The degree to which a person } \\
\text { experiences the world as } \\
\text { threatening and beyond his/her } \\
\text { control. }\end{array}$ \\
\hline
\end{tabular}

Source: Hogan and Hogan (2007).

\section{Measure of information: ScreenInfo and GuessSimilar}

To capture the value of the information that the subject saw on the screen, I introduce two indices: the index ScreenInfo is a measure of similarity of all the results that the subject saw on the screen and GuessSimilar is the measure of the similarity of the subject's original estimate to the observed values. ScreenInfo was computed with a simple approach: with the exception of zero, for all pairs of values available on the screen, when two values did not differ by more than one, the index got one point and the summation over all points created the index. The idea is that the more information on the screen, the higher probability for the subject to switch from her original estimate. GuessSimilar was computed in a similar fashion: if the subject's original estimate was not further than one from a value of an estimate on the screen, GuessSimilar got one point. Again, summation over all observed values yields the final value of GuessSimilar. The meaning is that the more similar one's guess to others' guesses is, the less meaningful it is to switch. 


\section{Risk attitudes}

The risk-averse subjects should suffer from a deterioration of performance under time pressure as in Cadsby et al. (2009) and the elevated levels of cortisol stemming from stressful situation may actually promote risk-taking as in van den Bos et al. (2009) or Porcelli and Delgado (2009). Therefore I control for this possible effect by measuring the risk-preferences of subjects by a standard risk-protocol taken from Dohmen et al. (2009). Specifically I measure a certainty equivalent and include it into regression analysis as a continuous measure of risk-aversion together with a $0 / 1$ variable risk-averse, which indicates if the certainty equivalent is below the expected value of the lottery.

\section{Other personal characteristics: Female, SelfConfidence, TotalProfit}

Generally speaking, we can also expect that the subjects with a higher task-specific self-confidence will have lower incentives to look at the public information and if they do, they will be reluctant to conform to the majority. In this case the confidence scale ${ }^{8}$ is reversed so the effect of SelfConfidence is expected to be positive on both explained variables.

The endowment effect caused by the fact that the participants saw their earnings after each round was controlled for by adding total profit (variable TotalProfit) that the subject had already earned. Because I expect it to behave similarly to the general behaviour of wage-related variables; i.e. that it is likely to be log-normal, I transform it by using a natural logarithm so that the new variable InTotProf is normally distributed. Female is a dummy variable indicating a female subject and it is added to control for the gender effect found by Baddeley et al. (2010), that a female is more likely to herd.

\section{Main Findings}

From the Graph 1 it is visible that the percentage of people using the public information is higher in the High level of time pressure. There were generally less people willing to see the public information, but once they saw it, they would have a higher chance to use it than in the other lower levels of time pressure. However, standard F-test results in that the levels are insignificantly different from each other, also when compared pair-wise. ${ }^{9}$

8 Participants had to estimate their relative position in the distribution of pay-offs after the first two tasks: the question was: "in which percentile do you think you are? 1=Top 20\% to $5=$ Lowest 20\%".

9 P-value $=0.576$ 


\section{Graph 1}

Percentage of Choices when Participants Were Affected by the Information about the Decisions of Others (InfoUsed) Conditionally on Seeing the Public Information

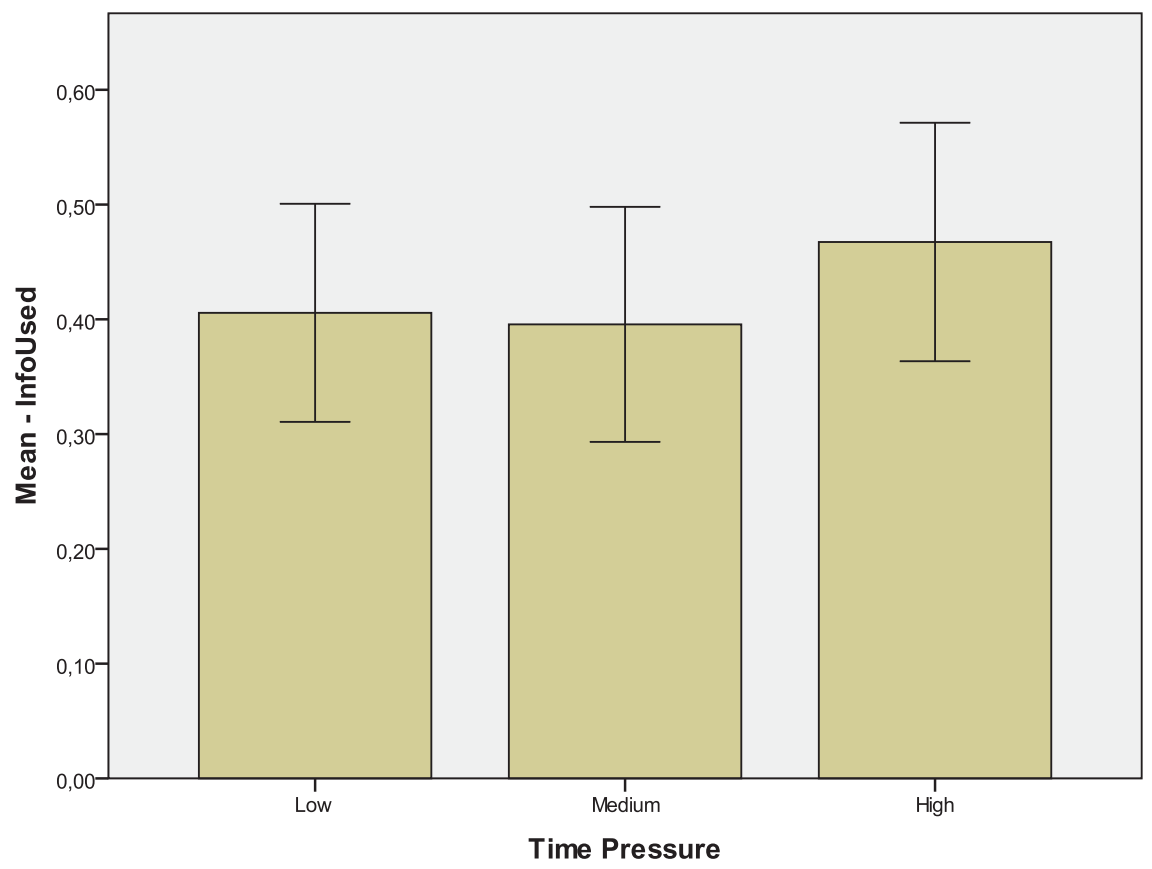

Error Bars: $95 . \% \mathrm{Cl}$

Source: own computations.

\section{Subjects' "Player" profiles}

In the experiment different types of subjects emerged: there were some that benefited from the possibility to see the public information, but also some for whom the information was useless. Out of 90 subjects, there were 13 subjects who never looked at the public info, and 8 out of them performed significantly better than average. This is the "successful" type of subject that would only lose money by viewing the public info. Apart from this, there was another type of subject who also never used the information, but this one must have had another motivation as their performance was mostly below average. I call this type "unsuccessful honest". ${ }^{10}$

On the one hand, there were 33 subjects who did look at the public info each time they had a chance to, but out of those 33 only five always used the information, so these "curious and imprecise" subjects were also not the only type of subjects. On the other hand, there were eight subjects who looked every time, but never switched - the "self-assuring"

10 I found out in feedback that there was a type of player not willing to see the public info due to fear of getting distracted by the results of others and thus performing even worse. 
types. These eight subjects were mostly highly successful in the task, so they probably just assured themselves that their result was correct.

Table 4

Descriptive Statistics of the Variables Used in the Model

\begin{tabular}{|l|c|c|c|c|c|c|}
\hline Variable & \multicolumn{1}{|c|}{ Explanation } & N & $\begin{array}{c}\text { Mini- } \\
\text { mum }\end{array}$ & $\begin{array}{c}\text { Maxi- } \\
\text { mum }\end{array}$ & Mean & $\begin{array}{c}\text { Std. } \\
\text { Deviation }\end{array}$ \\
\hline InfoUsed & If really used the info & 289 & 0 & 1 & 0.42 & 0.49 \\
\hline ScreenInfo & $\begin{array}{c}\text { Score of similarity of others' } \\
\text { values among themselves }\end{array}$ & 942 & 0 & 74 & 6.37 & 11.08 \\
\hline GuessSimilar & $\begin{array}{c}\text { Score of similarity of own } \\
\text { estimate to the others' values }\end{array}$ & 495 & 1 & 15 & 3.27 & 2.71 \\
\hline TimeDeciding & $\begin{array}{c}\text { Time spent on screen with } \\
\text { public information }\end{array}$ & 942 & 0 & 67.38 & 3.34 & 6.72 \\
\hline TimeLeft & $\begin{array}{c}\text { Time left when original } \\
\text { estimate set }\end{array}$ & 760 & 0 & 157 & 43.67 & 32.44 \\
\hline TP_Medium & Medium Time Pressure & 760 & 0 & 1 & 0.33 & 0.47 \\
\hline TP_High & High Time Pressure & 760 & 0 & 1 & 0.34 & 0.47 \\
\hline $\boldsymbol{O}$ & Openness to Experience & 942 & -4 & 20 & 9.99 & 5.22 \\
\hline C & Conscientiousness & 942 & -8 & 16 & 3.97 & 5.38 \\
\hline E & Extraversion & 942 & -13 & 18 & 2.83 & 6.64 \\
\hline A & Agreeableness & 942 & -6 & 18 & 4.57 & 4.67 \\
\hline $\boldsymbol{N}$ & Neuroticism & 942 & -20 & 8 & -4.17 & 5.16 \\
\hline SubjectiveStress & Stress (Subjective) & 760 & 1 & 10 & 5.76 & 2.45 \\
\hline Female & Female if 1 & 942 & 0 & 1 & 0.62 & 0.49 \\
\hline $\begin{array}{l}\text { Certainty } \\
\text { Equivalent }\end{array}$ & Certainty equivalent & 864 & 2 & 21 & 14.68 & 3.42 \\
\hline RiskAverse & Weakly Risk Averse & 942 & 0 & 1 & 0.92 & 0.28 \\
\hline SelfConfidence & Self Confidence & 942 & 1 & 5 & 3.16 & 1.22 \\
\hline TotalProfit & Difference of base-line to & 677 & 0 & 53 & 16.47 & 9.82 \\
\hline ObjectiveStress & \begin{tabular}{c} 
actual HR \\
\hline Sort
\end{tabular} & 942 & 0 & 2017 & 347.54 & 397.71 \\
\hline
\end{tabular}

Source: own computations.

\section{Was public information useful?}

We can have a look at the rate of "success" of revision: if the new estimate brought a higher pay-off than the original one. The percentage of successful changes is shown in Graph 2 -we can see that in most experimental sessions the subjects could improve using public the information in more than $80 \%$ cases. However, session No. 3 was exceptional and had this rate lower than $50 \%$. 


\section{Graph 2}

Rate of Success of Switching the Estimate after Seeing Public Information

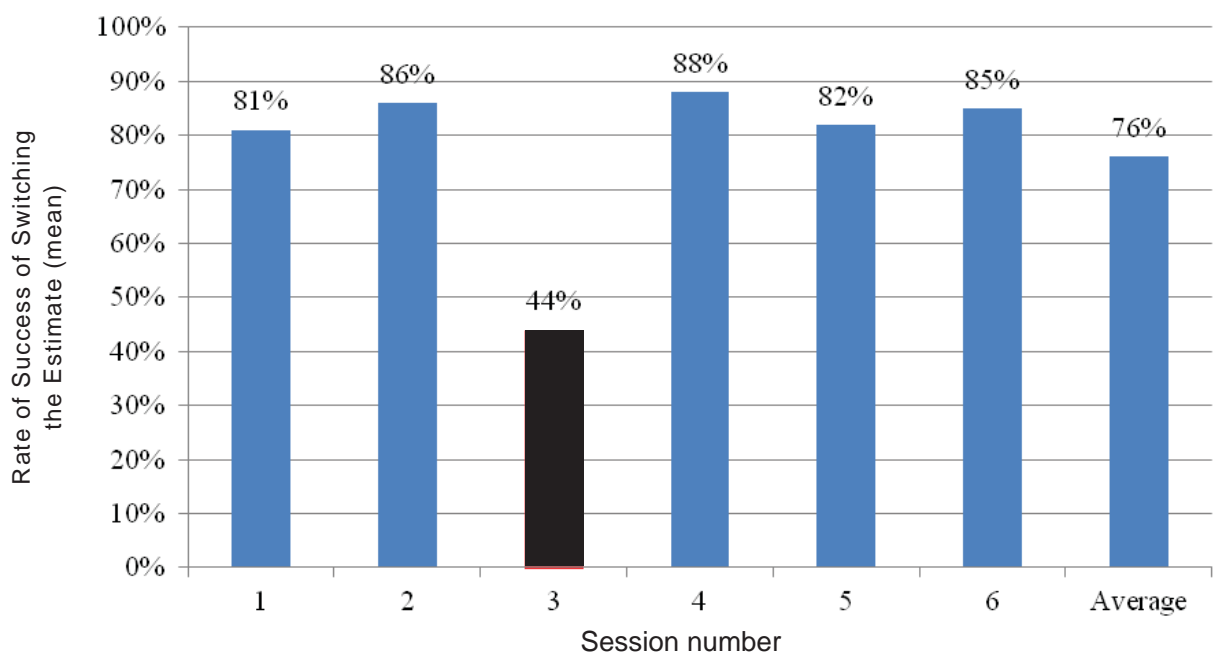

Source: own computations.

In this exceptional session No. 3 there were four subjects who randomly guessed the number shortly after the beginning of each task, so they added significant noise to the information seen on the screen by other subjects. Interestingly, their results were in the first three periods followed by others. As a result, the rate of successful switch in this session was much lower than in the other sessions where there were on average 3 incorrect switches, but in this session there were 14 incorrect switches. There were even incorrect cascades when the number followed was far from the true one: it happened in the first part of a period and it was caused by the subjects who guessed the result who were followed by two to three other subjects. However, in the second half of the period, three to four "honest" participants arrived and brought the correct information to light. Then the next subjects mostly either entered the result correctly or did not use the public info at all. This result strongly supports the fragility of cascades in a continuous setting: an incorrect cascade began, but was overrun by the arrival of the information brought by the subjects who counted well and their estimate was more precise. In real life, we also cannot distinguish who, when in a cascade, ignores private information and follows the crowd and on the contrary, who accidentally gets the same result and gets into a cluster of subjects with the same results. The results generally suggest that if subjects expect the arrival of true information to the public, moment of the arrival may, with a high probability, break the cascade.

\section{Time Pressure and Information Cascades / Herding}

The rate of revision from the original estimates was independent of time pressure (see Graph 1). This obviously goes against Hypothesis 1 and the underlying explanatory 
mechanism of Rieskamp and Hoffrage (2008) who suggest that if people have to work under increasing time pressure, they select faster a smaller amount of information that they consider to be worth it; i.e. they prefer more quality over quantity than in the treatment without time pressure.

\subsection{Data from heart-rate monitors}

I measured the average heart rate ${ }^{11}$ over the time when was the task performed (variable $H R \_A V G$ ); the base-line $\mathrm{HR}^{12}$ (var. $H R \_C A L M$ ) and the resulting difference between these two (ObjectiveStress), which should account for the personal physiological differences of different base-line HR levels. The summary statistics of the HR-variables are shown in Table 5. Some subjects had average HR almost the same as when they stayed calm in the end, others had peaks as high as 151 beats per minute, which is equivalent to a highly demanding physical activity.

Table 5

Descriptive Statistics of Variables HR_AVG, HR_CALM and ObjectiveStress

\begin{tabular}{|l|c|c|c|c|c|c|}
\hline & N & Min & Max & Mean & SE (Mean) & Std. Dev. \\
\hline $\begin{array}{l}\text { Average heart-rate } \\
\text { during the task }\end{array}$ & 677 & 59 & 151 & 90.94 & 0.601 & 15.634 \\
\hline Base-line Heart Rate & 677 & 50 & 98 & 74.47 & 0.391 & 10.179 \\
\hline $\begin{array}{l}\text { Difference of base-line } \\
\text { to actual HR }\end{array}$ & 677 & 0 & 53 & $\mathbf{1 6 . 4 7}$ & 0.377 & 9.816 \\
\hline
\end{tabular}

Source: own computations.

\section{Qualitative analysis}

Generally speaking, there were different kinds of curves of HR: a majority of them (over 50\%) were very legible and fit well to the data, i.e. there was a significant and stable increase during the performance of the task and the HR went back to normal levels between the tasks; but some were more or less random and similar to white noise. Interestingly, some subjects had a steep peak when they decided to guess the number instead of performing the task (took only a short time of thinking), but others did not. Many subjects also had a short peak just before a task started and then the normal hump-shape followed, which is a sign of a reaction to the introduction screen of each task. Overall, the HR during tasks was significantly different to the baseline rate, which proves the first part of the Hypothesis 2 on $1 \%$ level.

11 Further on labelled HR.

12 HR measured in a "steady" state when no activity is performed; the interval after completion of a questionnaire and before collecting the money. Also sometimes referred to as "quiescent". 
Table 6

Pearson Correlations. Note: $\left(^{*}\right)$ and $\left({ }^{* *}\right)$ Indicate Significance on $5 \%$ and $1 \%$ Levels Respectively

\begin{tabular}{|l|c|c|}
\hline \multicolumn{2}{|c|}{} & ObjectiveStress \\
\hline \multirow{3}{*}{ SubjectiveStress } & Pearson Correlation & $.105\left(^{*}\right)$ \\
\cline { 2 - 3 } & Sig. (2-tailed) & 0.013 \\
\cline { 2 - 3 } & $\mathrm{N}$ & 559 \\
\hline \multirow{4}{*}{ SelfConfidence } & Pearson Correlation & $.152\left(^{* *}\right)$ \\
\cline { 2 - 3 } & Sig. (2-tailed) & 0.000 \\
\cline { 2 - 3 } InfoUsed & $\mathrm{N}$ & 677 \\
\hline \multirow{3}{*}{} & Pearson Correlation & $\mathbf{- . 2 2 5 ( ^ { * * }}$ \\
\cline { 2 - 3 } & Sig. (2-tailed) & 0.001 \\
\cline { 2 - 3 } & $\mathrm{N}$ & 205 \\
\hline
\end{tabular}

Source: own computations.

Hypothesis 2 also stated that there should be a positive correlation between the objectively measured stress and subjectively stated levels of stress, in our case between variables SubjectiveStress and ObjectiveStress. In Table 6 you can see that indeed there is a significant positive relationship between the ObjectiveStress and subjective stress, but the level is rather very small. However, more interesting is the negative relationship between ObjectiveStress and the InfoUsed, which suggests that the more a person is in an aroused state (which may be a sign of stress, concentration or activity in general) the less willing she is to use the public information. Unfortunately, without another measure of stress it cannot be distinguished, what the reason for the increase of the HR was. ${ }^{13}$

\section{Stress and Risk Preferences}

An important part of the analysis is comparison of the levels of both subjective and physiological stress with respect to the risk attitudes. Graph 4 shows that the means of ObjectiveStress are insignificantly different from each other for the risk-averse and risk-loving subjects. There were only about $20 \%$ of participants risk-seeking, so the standard errors are larger relative to the risk-averse participants. Hypothetically, if we there were an equal number of risk-seeking participants and the error bars would have a similar size as of the risk-averse group, there may potentially be an identifiable difference. Interestingly, the increase in heart-rate is higher for women, but only those who are risk-seeking.

13 Another measure of stress has been by the time of conducting the experiment financially not affordable. 


\section{Graph 4}

Comparison of Levels of Stress with Respect to Risk Attitudes (left) and Splitted by Gender (right). t-test for the Equality of Means Does not Reject the Null for ObjectiveStress on 10\% Level of Significance

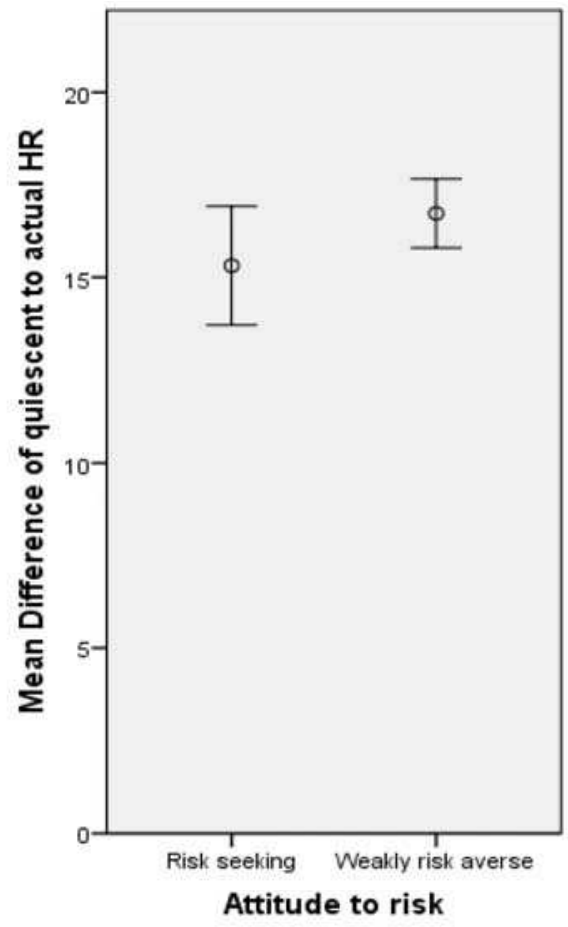

Error Bars: $95 \% \mathrm{Cl}$

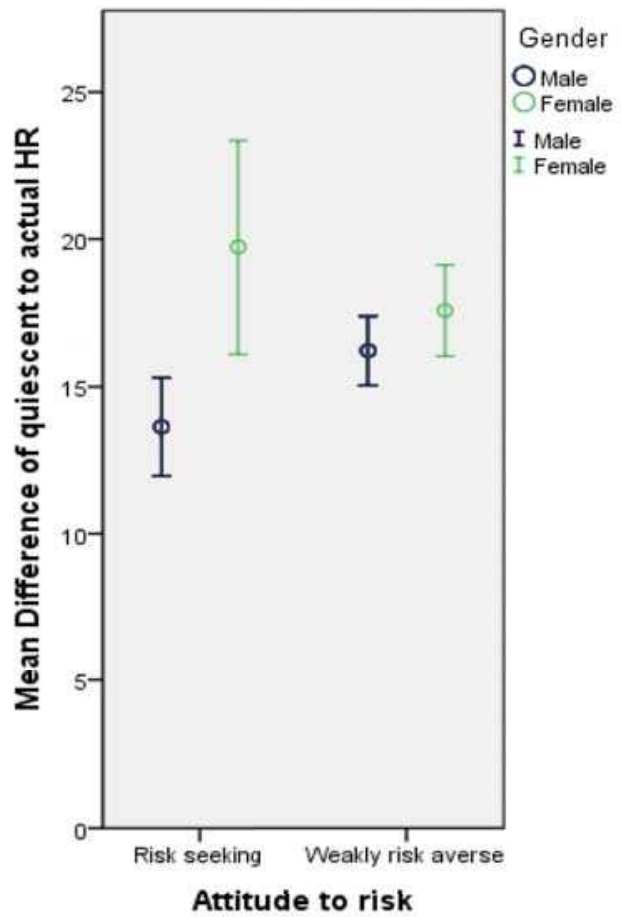

Error Bars: $95 \% \mathrm{Cl}$

\section{Stress and Time Pressure}

When we have a look on the differences in ObjectiveStress under the three levels of time pressure, we can see that under medium and high levels of time pressure the increase in heart-rate is significantly ${ }^{14}$ higher than in the low level, but the medium and high levels are not different. There are also no gender differences as shown in the right part of Graph 5 , although the levels are always slightly higher for women.

Graph 6 shows similar result for the subjectively reported level of stress. In the left part of the graph you can see that whereas the means of SubjectiveStress in Low and Medium levels of time pressure are insignificantly ${ }^{15}$ different from each other, in the High level of time pressure the mean is significantly higher than both of the other two

14 T-test for comparison of means of High and Low gives $p$-value $=0.054$ and comparison of Medium to Low gives $\mathrm{p}$-value $=0.05$.

15 T-test for comparison of means of High and Low as well as High and Medium gives p-value $=0.000$ and comparison of Medium to Low gives $\mathrm{p}$-value $=0.15$. 
levels. This proves that the participants were on average under higher subjective stress when facing the toughest time constraint. When we look at the gender differences in the same situation (right part of Graph 6), then there is an interesting result that females report higher level of stress than males, but the difference is significant only under the high level of time pressure, although in both low and medium levels of time pressure the subjective stress for women is slightly higher than for men.

\section{Graph 5}

Comparison of Levels of Stress with Respect to Risk Attitudes (left) and Splitted by Gender (right). t-test for the Equality of Means Does not Reject the Null for ObjectiveStress on 10\% Level of Significance

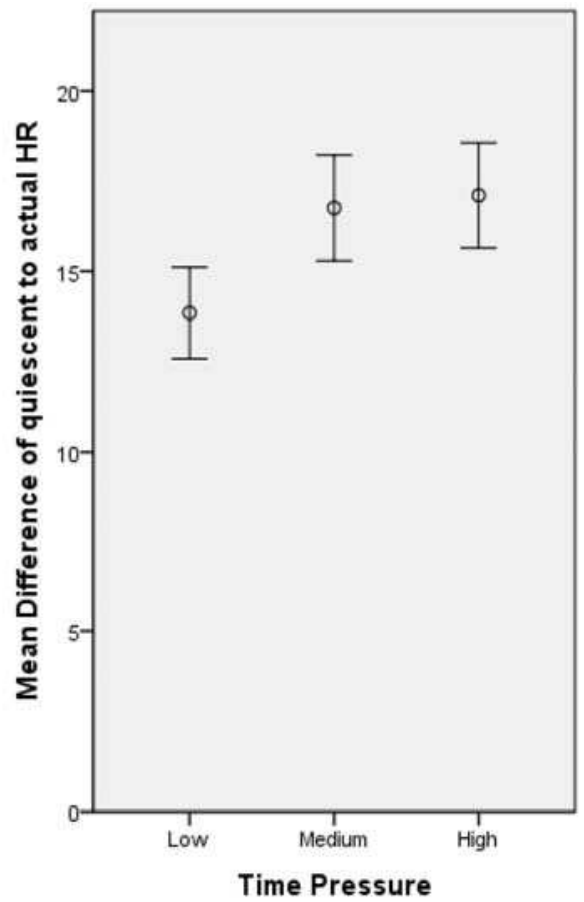

Error Bars: $95 \% \mathrm{Cl}$

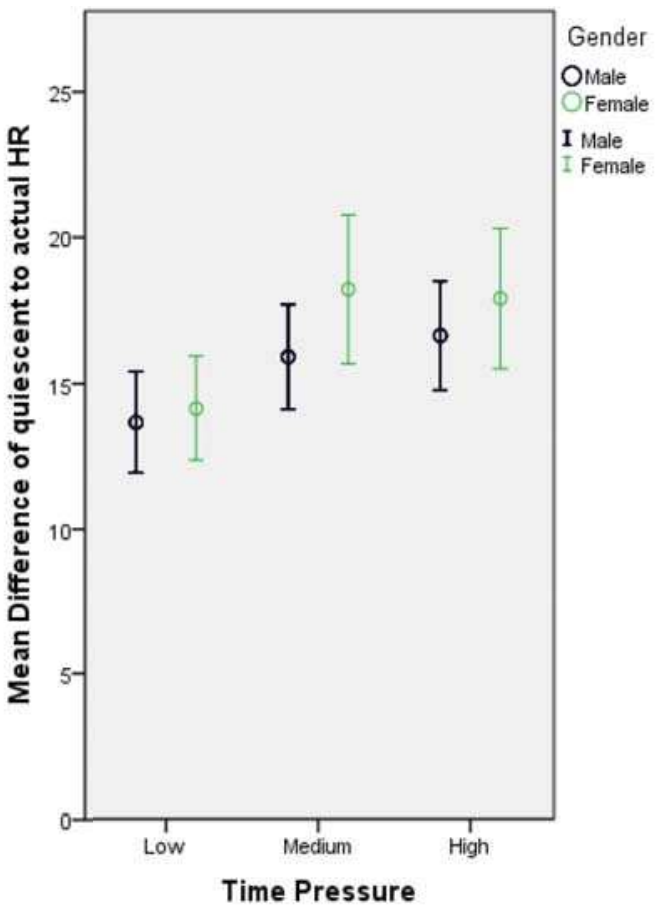

Error Bars: $95 \% \mathrm{Cl}$

Source: own computations. 


\section{Graph 6}

Comparison of Subjectively Stated Levels of Stress (variable SubjectiveStress) across Different Levels of Time Pressure (left) and also Gender Differences in Treatments (right)

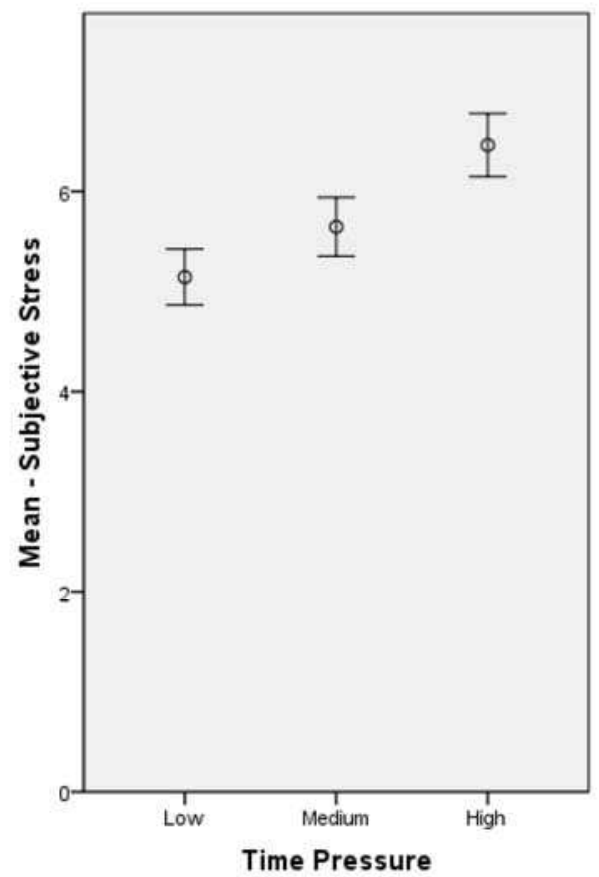

Error Bars: $95 \% \mathrm{Cl}$

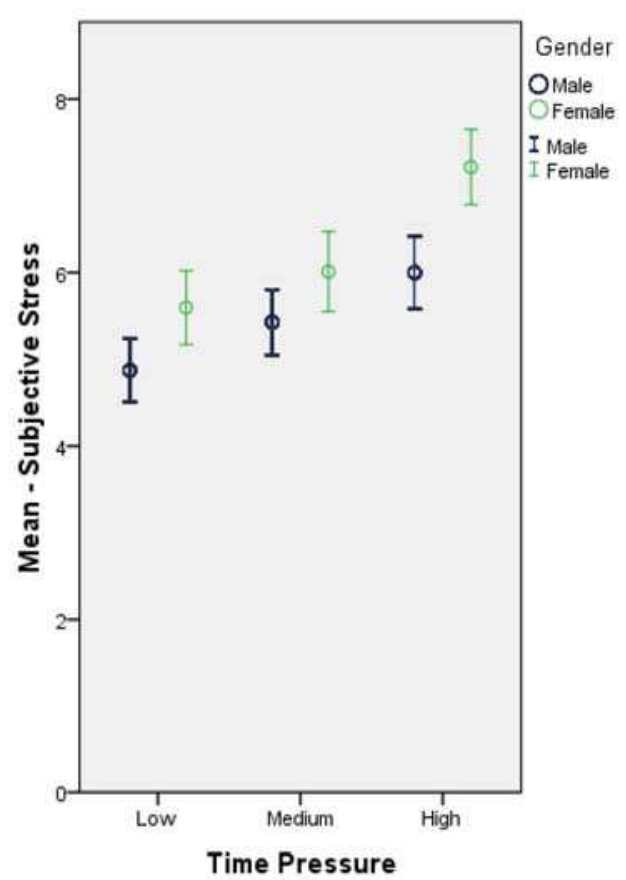

Error Bars: $95 \% \mathrm{Cl}$

\section{Model evaluation}

\subsection{Explaining the probability of using the public information - InfoUsed}

In Table 7 you can see summary of the regression results. Both variables indicating the information seen on the screen (ScreenInfo and GuessSimilar), the time subjects spent on the screen with the public information (TimeDeciding), personality traits extraversion and neuroticism (variables $E$ and $N$ ), and finally the log of total profit earned up to that time (lnTotProf) are significantly affecting the probability of switching. The insignificant variables were the dummies indicating the level of time pressure TP_Medium and TP_High, ObjectiveStress, ${ }^{16}$ both variables indicating subjects' risk attitudes, and the reported level of confidence. ${ }^{17}$ Furthermore, neither the total time left for the decision TimeLeft, nor Female influenced the probability of herding, which is contrary to the

16 Because the heart-rate monitors did not work for all subjects, I have a reduced number of observations in the regression when I include ObjectiveStress from 285 to 201. Therefore I perform a robustness check of significance of coefficients in the form of second regression equation where I omit this variable. You can see the results in the second column. They show that the results are fairly stable.

17 Remember, the scale is reversed - the higher number, the lower confidence. 
findings of Baddeley et al. (2010). The insignificance of both time-pressure dummies TP_Medium and TP_High means that I reject Hypothesis 1 as the probability of using public information was not significantly different in either of the levels of time pressure. However, there may have been an indirect effect of the level of time pressure through other variables, such as the public information provided on the screen could have been perceived as less valuable or the time to make the decision could have been perceived as too precious, which I cannot check with the present data.

Personality traits Neuroticism and Extraversion are significantly negatively associated with the probability of herding, which in case of Extraversion goes in line with Baddeley et al. (2010). In case of neuroticism the situation is not that clear, because Baddeley et al (2010) used a different set of personality traits. If I take as a counterpart of Neuroticism their Empathy, I can conclude that the result is similar in both studies, which is that the variable is significant and has a negative coefficient. These results suggest that an emotionally unstable and extraverted person is more likely to use the public information in his/her decision. Variable lnTotProf which is total profit earned up to the point of making a decision transformed by taking natural logarithm has in the regression significant and positive coefficient, which can be interpreted in a way that subjects with higher earned money were less cautious about the possible loss after changing their mind according to the public information.

The variables that generally indicated the time dimension of the task yield mixed results. Both dummies indicating the level of time pressure are not significant as well as the time the subjects had left to make a decision, but the time they spent on the screen with the public information is the variable with a the highest explanatory power and it has a positive relationship to the explained variable. The logic may thus be this: the subjects did have a look at the others' results, decided quickly whether they needed to change the coefficient or not, and then either left or started to think of the new value they should switch to, which was time consuming. Therefore, the causality may not be in the way that the longer time a subject stays, the more probable it is that she switches her estimate; but rather the opposite: if a subject wants to switch from her value, it will take her some time.

Both variables indicating information contained in the public screen, ScreenInfo and GuessSimilar, turn out to be steadily significant and thus it proves that the subjects behaved rationally in the sense that the additional information provided to them in this form influenced their decisions in the correct way. The positive sign of the coefficient of the ScreenInfo means that the more similar the coefficients of others, the more informative the screen was and thus the higher the probability of using the information. On the other hand, the negative sign of the GuessSimilar means that the more similar the subject's estimate to the estimates of the others' was, the lower the reason she had to change it. 
DOI: 10.18267/j.pep.468

Table 7

Logistic Model of InfoUsed

\begin{tabular}{|c|c|c|c|}
\hline \multicolumn{4}{|c|}{ Explained dependent variable: InfoUsed } \\
\hline & (1) & $(2)$ & (3) \\
\hline VARIABLES & Full model & ObjectiveStress excluded & TP excluded \\
\hline ScreenInfo & $\begin{array}{l}0.068^{\star *} \\
{[0.032]}\end{array}$ & $\begin{array}{l}0.064^{* *} \\
{[0.026]}\end{array}$ & $\begin{array}{c}0.048 \\
{[0.030]}\end{array}$ \\
\hline GuessSimilar & $\begin{array}{c}-0.506^{* * *} \\
{[0.187]}\end{array}$ & $\begin{array}{c}-0.531^{* * *} \\
{[0.136]}\end{array}$ & $\begin{array}{c}-0.502^{\star * *} \\
{[0.190]}\end{array}$ \\
\hline Time-Deciding & $\begin{array}{l}0.301^{* * *} \\
{[0.093]}\end{array}$ & $\begin{array}{c}0.237^{* * *} \\
{[0.056]}\end{array}$ & $\begin{array}{l}0.315^{\star * *} \\
{[0.098]}\end{array}$ \\
\hline TimeLeft & $\begin{array}{c}0.007 \\
{[0.015]}\end{array}$ & $\begin{array}{c}0.009 \\
{[0.011]}\end{array}$ & $\begin{array}{l}-0.005 \\
{[0.009]}\end{array}$ \\
\hline TP_Medium & $\begin{array}{l}-0.160 \\
{[0.710]}\end{array}$ & $\begin{array}{c}0.277 \\
{[0.449]} \\
\end{array}$ & \\
\hline TP_High & $\begin{array}{c}0.899 \\
{[0.757]}\end{array}$ & $\begin{array}{c}0.972 \\
{[0.610]}\end{array}$ & \\
\hline $\begin{array}{l}\text { Openness to } \\
\text { Experience }\end{array}$ & $\begin{array}{c}0.038 \\
{[0.047]}\end{array}$ & $\begin{array}{c}0.058 \\
{[0.038]}\end{array}$ & $\begin{array}{c}0.046 \\
{[0.048]}\end{array}$ \\
\hline Conscientiousness & $\begin{array}{c}-0.070 \\
{[0.052]}\end{array}$ & $\begin{array}{l}-0.049 \\
{[0.037]} \\
\end{array}$ & $\begin{array}{c}-0.062 \\
{[0.047]}\end{array}$ \\
\hline Extraversion & $\begin{array}{c}-0.151^{* * *} \\
{[0.055]}\end{array}$ & $\begin{array}{c}-0.128^{* * *} \\
{[0.038]}\end{array}$ & $\begin{array}{c}-0.164^{\star * \star} \\
{[0.055]}\end{array}$ \\
\hline Agreeableness & $\begin{array}{c}-0.021 \\
{[0.060]}\end{array}$ & $\begin{array}{c}0.040 \\
{[0.047]}\end{array}$ & $\begin{array}{c}-0.042 \\
{[0.059]}\end{array}$ \\
\hline Neuroticism & $\begin{array}{l}-0.115^{\star *} \\
{[0.057]}\end{array}$ & $\begin{array}{l}-0.080^{* *} \\
{[0.037]}\end{array}$ & $\begin{array}{l}-0.124^{\star *} \\
{[0.058]}\end{array}$ \\
\hline Subjective-Stress & $\begin{array}{l}-0.184^{*} \\
{[0.107]}\end{array}$ & $\begin{array}{l}-0.155^{\star *} \\
{[0.077]}\end{array}$ & $\begin{array}{c}-0.166 \\
{[0.105]} \\
\end{array}$ \\
\hline Female & $\begin{array}{c}0.253 \\
{[0.541]}\end{array}$ & $\begin{array}{c}0.009 \\
{[0.419]}\end{array}$ & $\begin{array}{c}0.313 \\
{[0.511]}\end{array}$ \\
\hline Certainty Equivalent & $\begin{array}{c}0.099 \\
{[0.095]} \\
\end{array}$ & $\begin{array}{l}-0.038 \\
{[0.062]} \\
\end{array}$ & $\begin{array}{c}0.124 \\
{[0.091]} \\
\end{array}$ \\
\hline RiskAverse & $\begin{array}{c}0.317 \\
{[0.617]}\end{array}$ & $\begin{array}{l}-0.736 \\
{[0.521]}\end{array}$ & $\begin{array}{c}0.478 \\
{[0.622]}\end{array}$ \\
\hline Self-Confidence & $\begin{array}{c}0.037 \\
{[0.239]} \\
\end{array}$ & $\begin{array}{l}0.274^{*} \\
{[0.147]}\end{array}$ & $\begin{array}{c}0.036 \\
{[0.232]} \\
\end{array}$ \\
\hline InTotProf & $\begin{array}{c}0.669^{* * *} \\
{[0.218]}\end{array}$ & $\begin{array}{c}0.536^{\star * *} \\
{[0.122]}\end{array}$ & $\begin{array}{c}0.637^{\star \star *} \\
{[0.185]}\end{array}$ \\
\hline ObjectiveStress & $\begin{array}{l}-0.024 \\
{[0.032]}\end{array}$ & & $\begin{array}{c}-0.014 \\
{[0.035]}\end{array}$ \\
\hline
\end{tabular}


(continuation)

\begin{tabular}{|l|c|c|c|}
\hline Constant & $\begin{array}{c}-6.643^{* *} \\
{[3.079]}\end{array}$ & $\begin{array}{c}-4.283^{* *} \\
{[2.093]}\end{array}$ & $\begin{array}{c}-6.415^{\star \star} \\
{[2.664]}\end{array}$ \\
\hline Observations & 201 & 285 & 201 \\
\hline Pseudo $\mathbf{R}^{2}$ & 0.463 & 0.409 & 0.455 \\
\hline Log-L & -72.49 & -114.6 & -73.68 \\
\hline Chi $^{2}$ & 59.03 & 87.21 & 51.13 \\
\hline
\end{tabular}

Note: Robust standard errors in parentheses. * , ** and ${ }^{* * *}$ indicate significance on $10 \%, 5 \%$ and $1 \%$ level, respectively. Source: own computations.

\subsection{Hypothesis evaluation}

\section{Hypothesis 1 - Herding and time pressure}

Both dummy variables indicating the time pressure are not significantly different from zero, and this result is fairly stable across various specifications, so we can conclude that there is no general relationship between the level of time-pressure and probability to herd. On the other hand, the time dimension played an important role in the time they spent looking at the public information - and both must have been implicitly influenced by the total available time that varied with the level of time pressure.

\section{Hypothesis 2 - Stress and heart rate}

The result is that the average difference of the heart rate during the task to the base level was 16.47 so the variable ObjectiveStress looks like a good measure of the induced stress, if it is positively correlated with subjectively stated level of stress. The heart rate of some subjects was overall not different to white noise, but the majority had very clearly identifiable periods of performance in comparison to the base level with some subjects reaching as high as 150 beats per minute. As it is shown in the Table 6, the correlation of ObjectiveStress and SubjectiveStress is indeed positive and significant on a 5\% level, but it is rather small - only 0.1 . This shows a discrepancy between the reported and revealed/ direct measure of stress which is probably due to the fact that the increase in heart-rate may signal a higher activity, but not necessarily stress.

\section{Conclusion}

The main purpose of this paper was to explain the individual propensity to herd with a special concern to the effect of time pressure. To do this, I designed and carried out a laboratory experiment where I measured occurrence of herding as a $0 / 1$ variable when participants changed their original decision after being exposed to information about the decisions of others. Apart from the behaviour in the task, I also tracked individual attributes such as risk attitude, task-specific confidence, personality traits and subjective 
as well as "objective" levels of stress. The most important result of this experiment is that time pressure indicated by a set of three $0 / 1$ indicator variables played no significant role in the model of herding. Nevertheless, the time dimension, as revealed by the time spent on the screen with public information, was significant and important and thus the time pressure needs to be further examined by using a finer resolution. Apart from that, both variables capturing the information contained in the others' estimates were significant, behave as expected and have a considerable predictive power. Another important predictor is the transformed total profit the subjects had acquired until the point of making the decision and its effect was that the more they had earned, the higher was the probability they would herd. Personality traits Neuroticism and Extroversion were significantly negatively associated with probability to herd, which goes in line with other literature and which can be interpreted in the way that an emotionally unstable extroverted person has a higher probability to mimic behaviour of others. Subjectively perceived stress was correlated to the objectively measured indicator of heart-rate increase during a task, but the correlation was very weak which suggests that the heartrate increase may have indicated rather physical arousal in general than stress. Generally speaking, even though the results from this experiment have to be treated with care due to the specific nature of the given task, this experiment has provided an insight into the state of the analysis of behaviour under time pressure, especially in connection to the propensity to herd. However, more research in this area is needed.

\section{References}

Baddeley, M., Burke, C., Schultz, W., Tobler, T. (2010), "Impacts of Personality on Herding in Financial Decision-Making." Cambridge Working Papers in Economics No. 1006.

Baddeley, M. C., Pillas, D., Christopoulos, Y., Schultz, W., Tobler, P. (2007), "Herding And Social Pressure In Trading Tasks: A Behavioural Analysis." Cambridge Working Papers in Economics, No. 730.

Banerjee, A. V. (1992), "A Simple Model of Herd Behavior." Quarterly Journal of Economics, Vol. 107, pp. 797-817.

Bikhchandani, S., Hirshleifer, D., Welch, I. (1992), "A Theory of Fads, Fashion, Custom and Cultural-Change as Informational Cascades." Journal of Political Economy, Vol. 100, pp. 992-1026.

Brock, W. A., Durlauf, S. N. (2000), "Interactions Based Models," NBER Technical Working Paper No. 258, Cambridge MA: National Bureau of Economic Research.

Cadsby, C. B., Song, F., Tapon, F. (2009), "The Impact of Risk Aversion and Stress on the Incentive Effect of Performance Pay." University of Guelph Working Paper Series, No. 912.

Cameron, A. C., Trivedi, P. (2010), "Microeconometrics Using Stata," Revised Edition. StataCorp LP.

Cao, H. H., Hirshleifer, D. A. (2000), "Conversation, Observational Learning, and Informational Cascades." Dice Center Working Paper No. 2001-5.

Dohmen, T., Falk, A., Huffman, D., Sunde, U., Schupp, J., Wagner, G. G. (2009), "Individual Risk Attitudes: Measurement, Determinants and Behavioral Consequences." Research memoranda, Maastricht: METEOR, Maastricht Research School of Economics of Technology and Organization, No. 39.

Falk, A., Menrath, Kupio, Siegrist (2006), "Unfair Pay and Stress." mimeo. 
Fischbacher, U. (2007), "Z-TREE: Zurich Toolbox for Ready-made Economic Experiments." Experimental Economics, Vol. 10, pp. 171-178.

Gilbert, T., Kogan, S. (2005), "Learning From the Skills of Others: Experimental Evidence." GSIA Working Papers No. 25.

Goldberg, L. R. (2010), "International Personality Item Pool: A Scientific Collaboratory for the Development of Advanced Measures of Personality Traits and Other Individual Differences." [Online]. Available at http://ipip.ori.org/.

Hogan, R., Hogan, J. (2007). "Hogan Personality Inventory Manual, Third Edition" Tulsa, OK, Hogan Assessment Systems.

Kemeny, M. E. (2003), "The Psychobiology of Stress." Current Directions in Psychological Science, Vol. 12, pp. 124-129.

Kirschbaum, C., Pirke, K. M., Hellhammer, D. H. (1993), "The 'Trier Social Stress Test' - A Tool for Investigating Psychobiological Stress Responses in a Laboratory Setting." Neuropsychobiology, Vol. 28, pp. 76-81.

Kocher, M. G., Sutter, M. (2006), "Time Is Money - Time Pressure, Incentives, and the Quality of Decision-making." Journal of Economic Behavior \& Organization, Vol. 61, pp. 375-392.

Lo, A. W., Repin, D. V. (2001), "The Psychophysiology of Real-Time Financial Risk Processing." National Bureau of Economic Research Working Paper Series, No. 8508.

Lundberg, U. (ed.) (1993), On the Psychobiology of Stress and Health. New York: Springer.

Maule, J. A., Edland, A. C. (2000), "The Effects of Time Pressure on Human Judgment and Decision Making," in Ranyard, R., Crozier, W. R., Svenson, O., eds., Decision Making: Cognitive Models and Explanations. London: Routledge.

Payne, J. W., Bettman, J. R., Luce, M. F. (1996), When Time Is Money: Decision Behavior under Opportunity Cost Time Pressure. Kidlington, ROYAUME-UNI: Elsevier.

Porcelli, A. J., Delgado, M. R. (2009), "Acute Stress Modulates Risk Taking in Financial Decision Making." Psychological Science, Vol. 20, pp. 278-283.

Rieskamp, J., Hoffrage, U. (2008), "Inferences under Time Pressure: How Opportunity Costs Affect Strategy Selection." Acta Psychologica, Vol. 128, pp. 258-276.

Svenson, O., Benson, L. (eds.) (1993), On Experimental Instructions and the Inducement of Time Pressure Behavior. New York: Springer.

Van Den Bos, R., Harteveld, M., Stoop, H. (2009), "Stress and Decision-making in Humans: Performance Is Related to Cortisol Reactivity, Albeit Differently in Men and Women." Psychoneuroendocrinology, Vol. 34, pp. 1449-1458.

Welch, I. (1992), "Sequential Sales, Learning and Cascades." Journal of Finance, Vol. 47, pp. 695-732. 